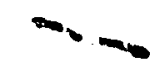

NATIONAL AERONAUTICS AND SPACE ADMINISTRATION

Technical Memorandum 33-694

\title{
A Shock Spectra and Impedance Method To Determine a Bound for Spacecraft Structural Loads
}

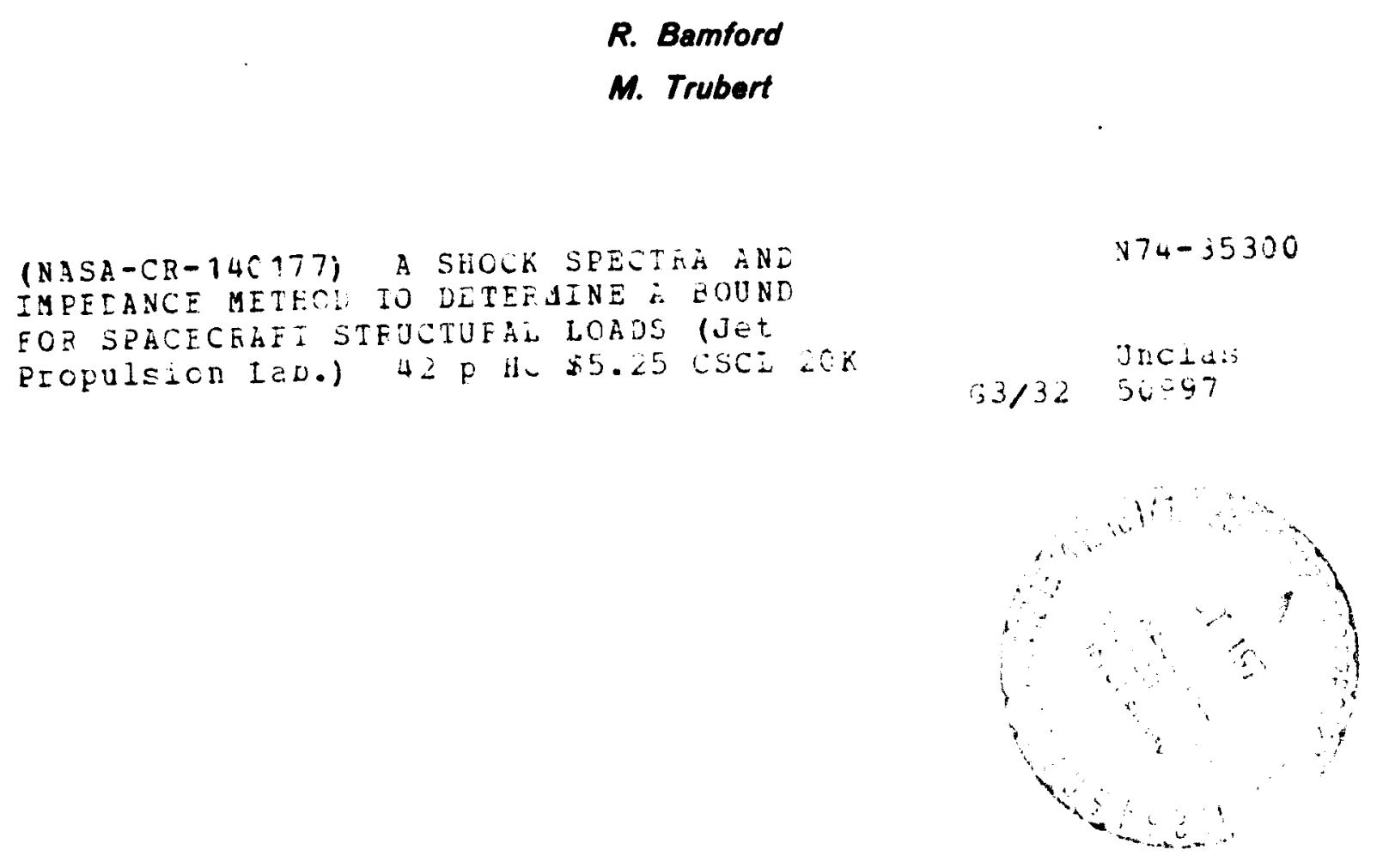

JET PROPULBION LABORATORY CALIFORNIA INSTITUTE OF TECMNOLOEY

pasadema, califonmia

septe bor 1. 1974 


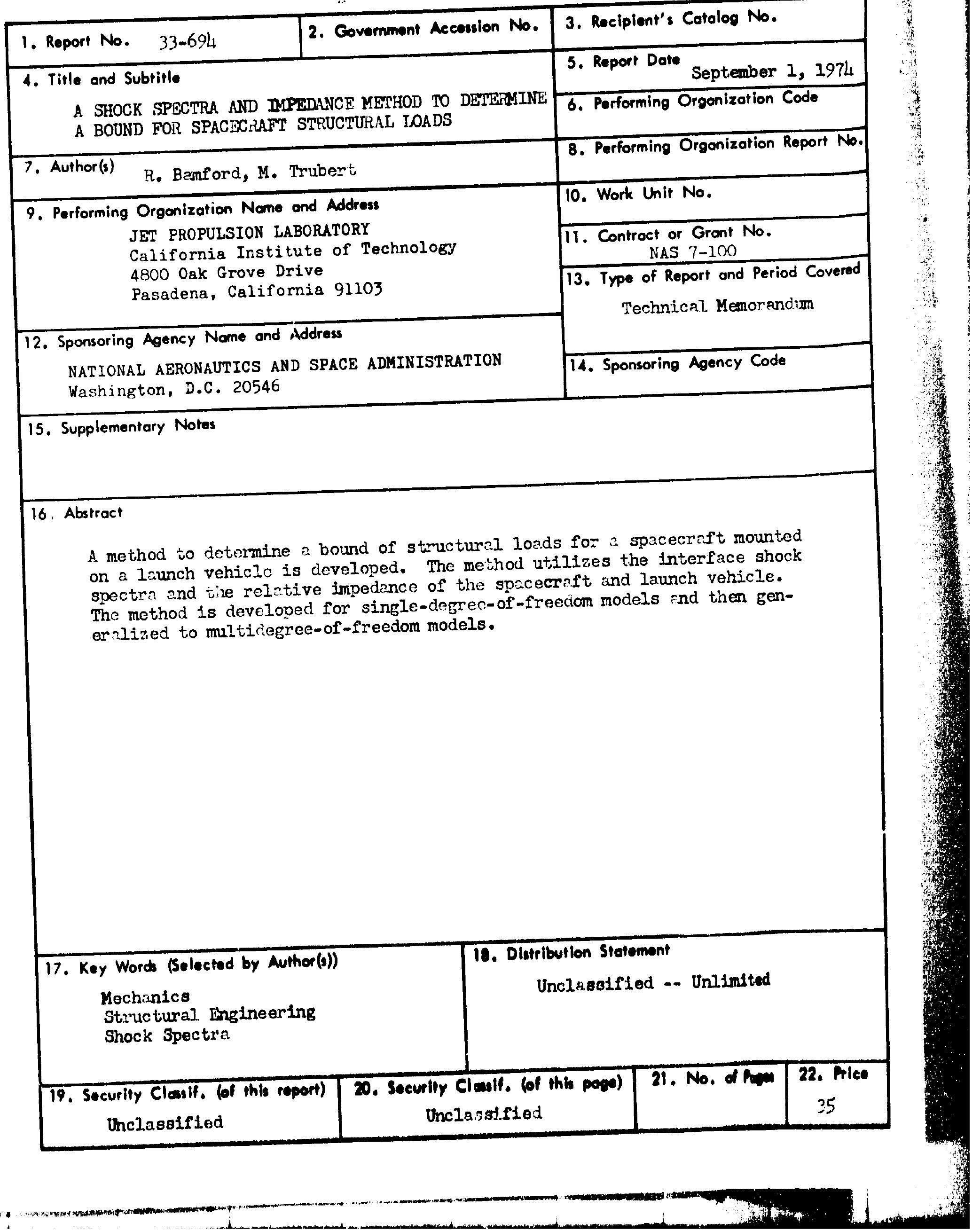


Make items 1, 4, 5, 9, 12, and 13 ogree with the corresponding information on the report cover. Use all capital letters for title (item 4). Leave items 2, 6, and 14 blank. Complete the remaining items as follows:

3. Recipient's Catalog No. Reserved for use by report recipients.

7. Author(s). Include corresponding information from the report cover. In addition, list the offiliation of an author if it differs from that of the performing organization.

8. Performing Organization Report No. Insert if performing organization wishes to assign this number.

10. Work Unit No. Use the agency-wide code (for exomple, 923-50-10-06-72), which uniquely identifies the work unit under which the woik was outhorized. Non-NASA performing organizalions will leave this blank.

11. Insert the number of the contec $t$ or grant under which the report was prepored.

15. Supplementary Notes. Enter information not included elsewhere but useful, such as: Prepared in cooperation with... Tronslation of (or by)... Presented at conference of... To be published in...

16. Abstract. Include a brief (not to exceed 200 words) factual summary of the most significant information contained in the report. If possible, the abstract of a classified report should be unclassified. If the report contains a significant bibliography or literature survey, mention it here.

17. Key Words. Insert terms or short phrases selected by the outhor that identify the principal subjects covered in the report, and thot are sufficiently specific and precise to be used for cataloging.

18. Distribution Statement. Enter one of the authorized statements used to denote releasability to the public or a limitation on dissemination for reasons other than security of defense information. Authorized statements are "Unclassified-Unlimited, "U. S. Government and Contractors only," "U. S. Government Agencies only, " and "NASA and NASA Controctors only."

9. Security Classification (of report). NOTE: Roports carrying a security classification will require additional markings giving security and downgrading information as specified by the Security Requirements Chacklist and the DoD Industrial Security Manual (DoD 5220.22-M).

20. Security Classificaition (of this page). NOTE: Becouse this poge may be used in preporing onnouncements, bibliographies, and data banks, it should be unclassified if possible. If a classification is required, indicate soporately the classification of the tille and the abstract by following these irems with either "(U)" for unclassified, or "(C)" or "(S)" as applicoble for classified irems.

21. No. of Poges. Insert the number of poges.

22. Price. Insert the price set by the Clearinghouse for Federal Scientific and Tect nical Information or the Government Printing Office, if known. 
NATIONAL AERONAUTICS AND SPACE ADMINISTRATION

\section{Technical Memorandum 33-694}

\section{A Shock Spectra and Impedance Method To Determine a Bound for Spacecraft Structural Loads}
R. Bamford
M. Trubert

JET PROPULSION LABORATORY caliFonmia institute of teommoloer

pasadema, cabirogmia

Septomber 1, 1974 


\section{PREFACE}

The work described in this report was performed by the Applied Mechanics Division of the Jet Propulsion Laboratory. 


\section{CONTENTS}

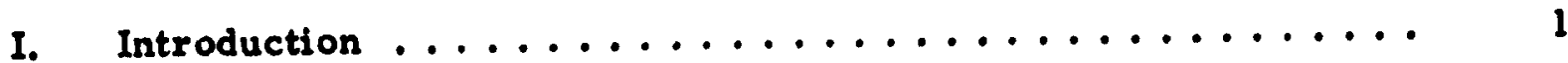

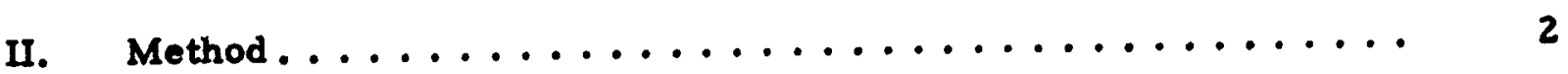

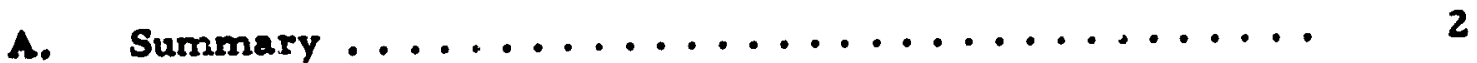

B. Shock spectra ...................... 6

C. Use of the Shock Spectra ................. 8

D. Determination of the Reduction Factor ............ 9

E. Generalization ....................... 19

References ............................ 27

\section{APPENDIXES}

A. Variation of Interface Acceleration in Terms of Spacecraft/Launch Vehicle Interaction...........

B. Conservative Replacement for

Relative Displacement .................

FIGURES

1. Shock spectrum oscillator ................. 6

2. Shock spectra and envelope ................ 7

3. Composite structure .................... 9

4. Unloaded launch vehicle .................. 9

5. Hypothetical curve enveloping peaks of shock spectra ........................ 1

6. Infinitesimal osclllator on unloaded interface ....... 12

7. Launch vehicle/ spacecraft composite structure....... 13

8. Modal model of composite etructure .............. 14

9. Relative velocitied for epacecraft cantilevered modes .................... 15

10. Response reduction due to relative impedance ....... 18 
11. Shock spectra peak sharpnese for top of Centaur stub adaptor .................... 18

12. Shock spectra envelopes at top of Titan III-C ....... 20

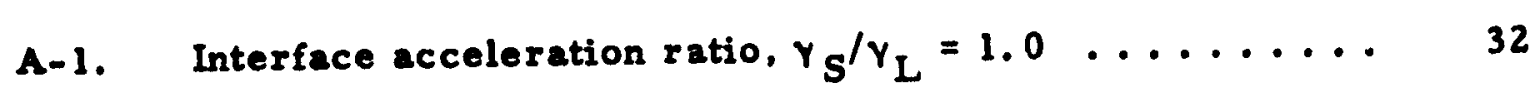

A-2. Interface acceleration ratio, $\bar{\gamma}=0.02 \ldots \ldots \ldots \ldots$

A-3. Interface acceleration ratio, $r=\left(\omega \frac{k}{s} / \omega_{L}^{i}\right)=1.0 \ldots 33$ 


\begin{abstract}
A method to determine a bound of structural loads for a spacecraft mounted on a launch vehicle is developed. The method utilizes the interface shock spectra and the relative impedance of the spacecraft and launch vehicle. The method is developed for single-degree-of-freedom models and then generalized to multidegree-of-freedom models.
\end{abstract}




\section{INTRODUCTION}

This report deals with the determination of structural design loads for a spacecraft mounied on a launch vehicle for which the range of significant resonant frequencies overlaps linat of the spacecraft. Several methods have been used in the past to obtain spacecraft structural design loads.

Analytically, the most elegant method to obtain loads has been to perform transiert analysis on a composite mathematical model of the spacecraft and launch vehicle. This requires the availability of forcing functions, for example, transient chamber pressure of the vehicle engines, that are applied to the composite model to compute accelerations and member loads for the entire composite structure. The main advantage of the transient analysis method is that it leads to a lighter-weight design. However, the method is costly, leads to a complicated ir... face, and the results are sensitive to changes in the structures. Fabrication tolerances prevent exact definition of the model, and it is impractical to analyze all possible combinations of extreme tolerances. When this approach is used, testing must be limited to the derived loads.

Another widely used procedure has been to use the shock spectra of - pacecraft/launch vehicle interface acceleration measured in previous flight to define an environment more hostile than the actial tiight. The envelope of the shock spectra is used for the definition of an equivalent sinusoidal spacecraft base acceleration. Design and test have been made for either base control or responce control. The advantage of this procedure is its simplicity, but the disadvantage is that it is sensitive to damping estimates, leads to heavy otructural weight, and may not alway be conservative.

This report proposes a method for the determination of deaign loads that are reallotic upper bounds of the flight loads. The method utilizes shock opectra of launch vehicle acceleration. in a rational manner and introduces the relative impedance of the spacecraft and launch vehtcle. It uses global modal properties wuch as rigid clastic mass coupling which are lese senoltive to minor modificatione than the modal displacement of a preselected degree of frsedom au for the traditional base acceleration approach. 
The main objectives sought in the method are low cost of analysis, timeliness within the design schedule and high reliability. These objectives are attained at on!y a moderate expense of structural weight compared to a transient analysis design. A major advantage of the method is that the interaction between spacecraft and launch vehicle is introduced in a simple nianner which permits a spacecraft loads analysis cycle to be performed without the necessity of a spacecraft/launch vehicle composite modal analy sis and flight simulation. Thus, the interface problems between the organi$z$ ations responsible for the spacecraft and the launch vehicle are greatly minimized. Other advantages are low sensitivities to the details of the mathematical model of the structures, to damping estimates, and to minor structural changes. The method is recommended when rapid convergence to final loads is more important than the weight saving possible from detuning of spacecraft and launch vehicle modes.

Finally, the method is a "follow on" method since it requires that spacecraft/i unch vehicle interface flight accelerations have been either measured or calculated for previous flight using the same launch vehicle.

\section{METHOD}

\section{A. SUMMARY \\ 1. Assumptions}

The assumptions on which the present method is based are:

(1) The spacecraft and launch vehicle are adequately represented by linear mathematical models.

(2) The shock spectra of the interface acceleration of previous flight: are available from which an envelope can be constructed. The method ts established for an unloaded interface, but if no data is available for an unloaded interface, it is assumed th th shock spectra for such an unloaded interface is within the envelope based on the loaded Interface data.

Response shock epectra of future flights will be within conservative envelope of those obtained from the prior flights. 
(4) The frequency distribution of the source of the disturbance is relatively flat compared to that of the interface acceleration response.

Note that most of the enumerated assumptions are also invoked for other methods of loads analysis.

\section{Procedure}

The method described here requires that the spacecraft to be analyzed and the launch vehicle be represented by their normal modes. Each spacecraft cantilever mode and launch vehicle mode having resonant frequencies in the same range are paired. Then the largest possible spacecraft modal response is determined by allowing artificial shift of the two resonant frequencies to produce "tuning" between the two modes.

Shock spectra of spacecraft/launch vehicle interface acceleration of previous flights are needed to define the basic load. The relative impedance between launch vehicle and spacecraft modes is utilized to determine a reduction factor $C$ of the dynamic response from that of a spacecraft with negligible inpedance, i.e., from the shock spectra $S^{\gamma}$.

The shock spectra is that of the unloaded interface (no spacecraft attached on the launch vehicle) and is assumed to have peaks, at the launch vehicle resonant frequencies, whose amplitudes are those of an envelope $S^{\gamma e}$ of the available shock spectra. This envelope $S^{y e}$ is assumed to enclose the shock spectra of the unloaded launch vehicle.

The interface reaction and the member loads are determined for all possible reasonable pairings, and the corresponding absolute values of the loads are combined. The combination also includes the quasi-static case, if present. 


\section{Terminology}

The romenclature used herein is presented as follows:

\section{(1) Symbols}

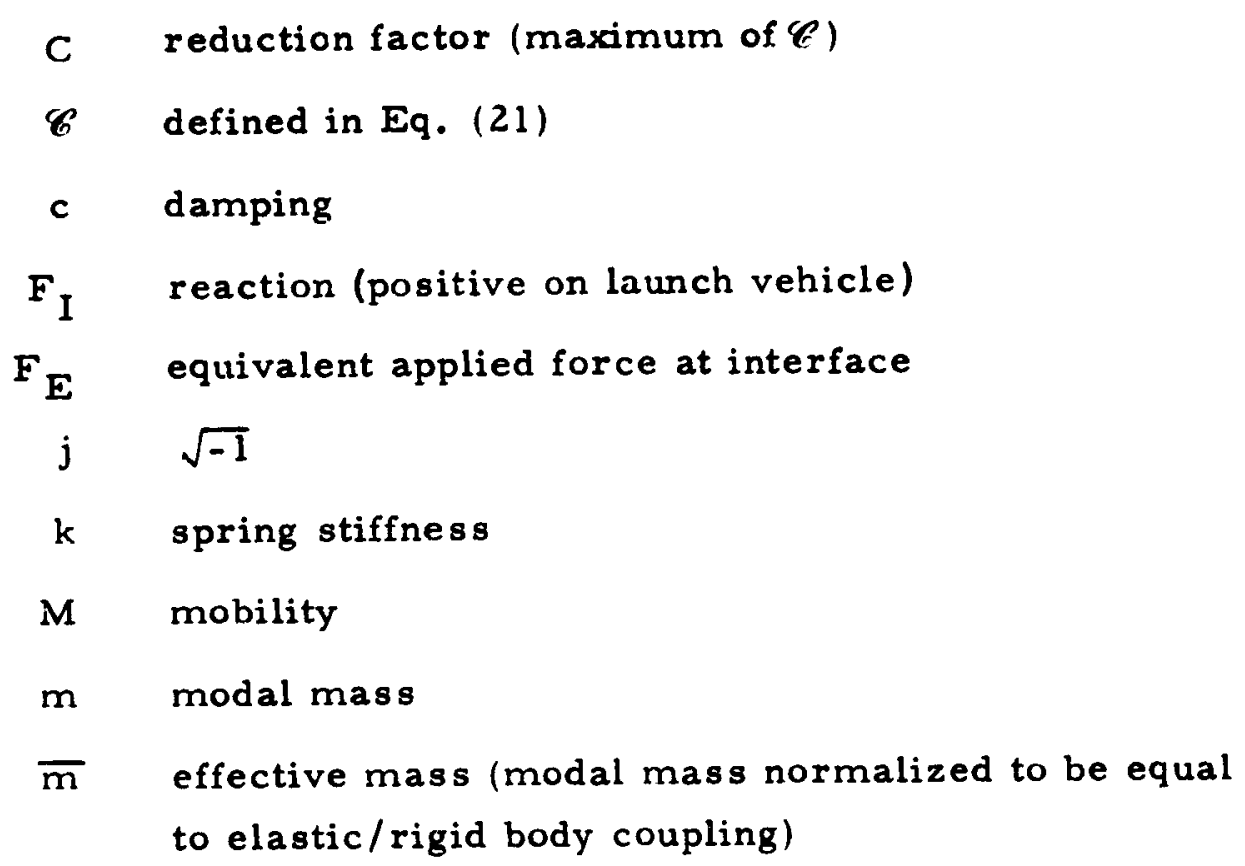




$$
\begin{array}{ll}
\omega & \text { circular frequency } \\
{[]} & \text { a rectangular matrix } \\
\{\} & \text { a vector }
\end{array}
$$

(2) Subscripts and Superscripts. Subscripts are used to indicate degrees of freedom both physical and generalized. Superscripts are used as mcdifiers of the symbol if any ambiguity remains after consideration of any subscripts.

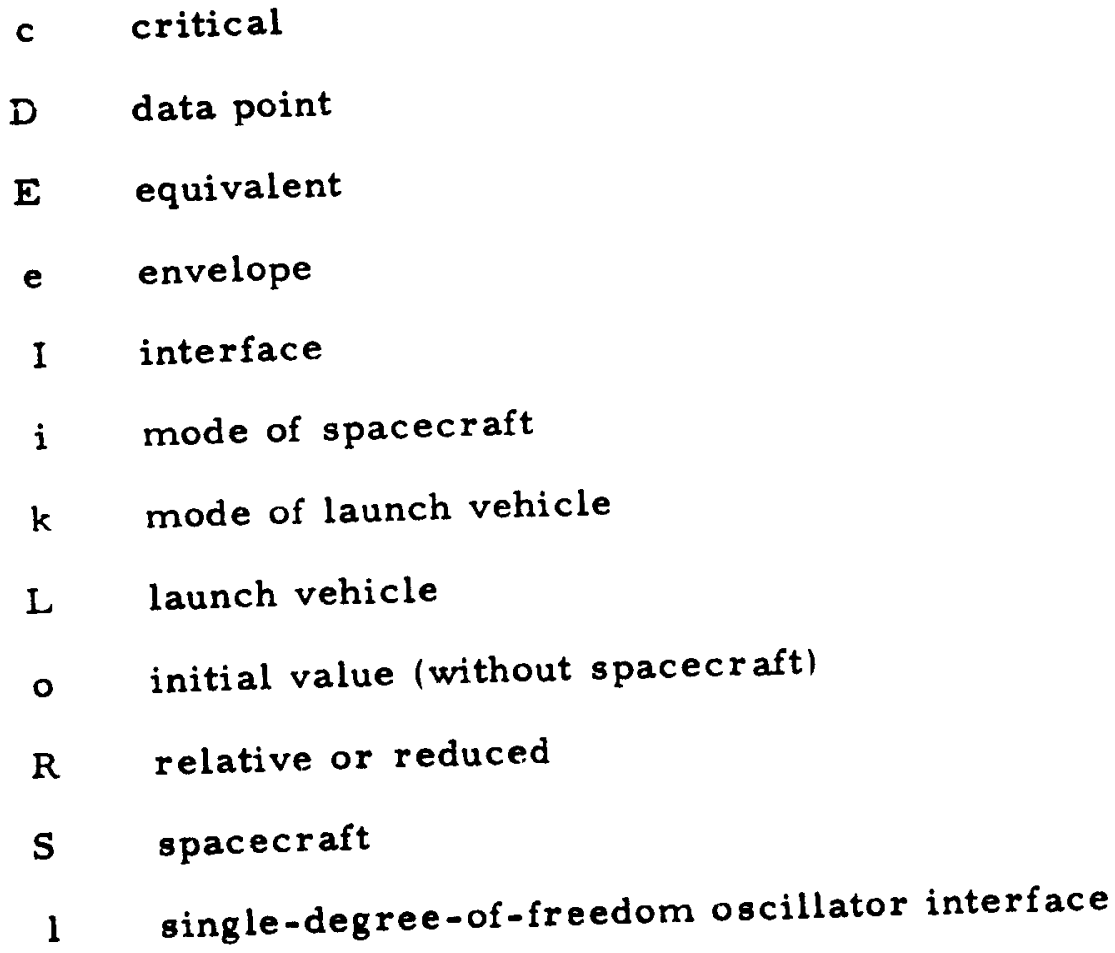

( ) ) derivative of ( ) with respect to time

$=$ equal

$\cong \quad$ approximately equal

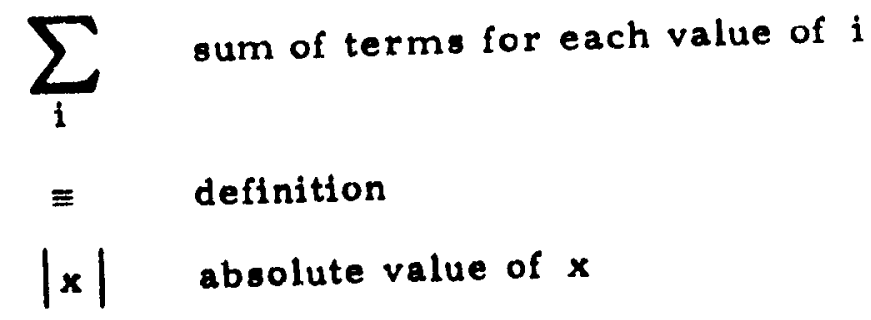




$$
\begin{array}{ll}
{[]^{T}} & \text { tranrpose of }[] \\
{[]^{-1}} & \text { inverse of }[]
\end{array}
$$

\section{B. SHOCK SPECTRA}

\section{Definition}

The shock spectrum $S^{\gamma}(\omega)$ is the maximum acceleration response of a single-degree-of-freedom oscillator of mass $\mathrm{m}$, damping $\gamma=2 \mathrm{c} / \mathrm{c} c$, and natural frequercy $\omega$ to a base acceleration $\ddot{u}_{1}^{\circ}(T)$ (Fig. 1 ).

$$
S^{\gamma}(\omega) \equiv\left|\ddot{u}_{R}(T)+\ddot{u}_{1}^{\circ}(T)\right|_{\max }
$$

where $i_{R}(T)$ is the acceleration of the mass $m$ relative to the base. Here the base is the spacecraft/launch vehicle interface.

It is noted that:

(1) The base acceleration $\ddot{u}_{1}^{\circ}(T)$ is an input unaffected by the presence of the oscillator; i.e., the impedance of the oscillator is infinitesimal compared to that of the base.

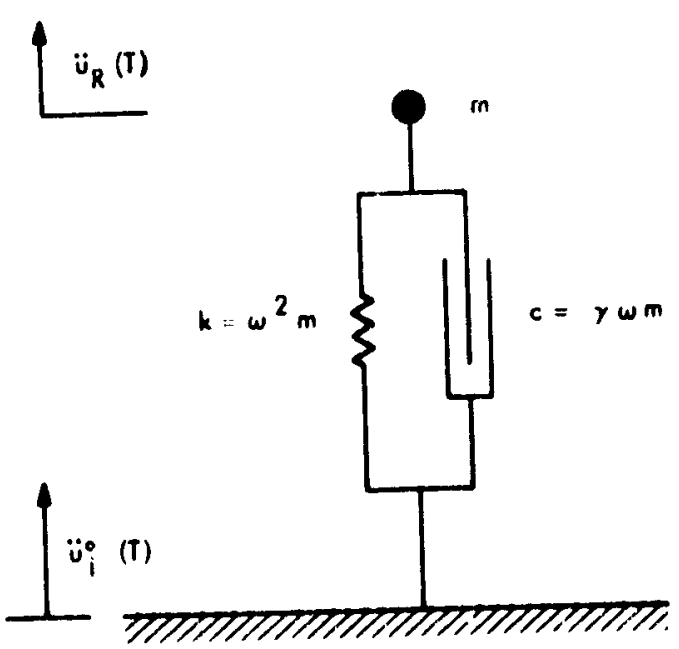

Fig. 1. Shock spectrum oscillator 
(2) The shock spectrum is a function of two variables, the natural frequency $\omega$ and the damping $y$ of the oscillator, and exhibits a series of peaks of various heights and sharpnesses.

The maximum reaction force $F_{1}^{0}$ on the spring connecting the mass $m$ to the base is equal to the inertia! force of the mass $\mathrm{m}$.

$$
F_{1}^{0}=m S^{\gamma}(\omega)
$$

Therefore, the shock spectrum is a measure of the reaction force on a unit mass. This observation is an essential part of the method presented in this report.

\section{Envelope}

Because in structural design one has to consider the most critical loading condition, it is customary to envelope the ensemble of shock spectra $\left[s^{\gamma}(\omega)\right]^{n}, n=1,2, \ldots, N$, of the interface acceleration $\left[\ddot{u}_{1}(T)\right]^{n}$ of previous flights, measured or calculated, using the same launch vehicle in order to determine a shock spectra envelope $S^{\gamma e}$ which is substituted for $S^{\gamma}(\omega)$. The construction of the envelope introduces a degree of conservatism that depends upon how it is done. Figure 2 shows the traditional enveloping technique by which straight lines are drawn tangent to the highest peaks of the

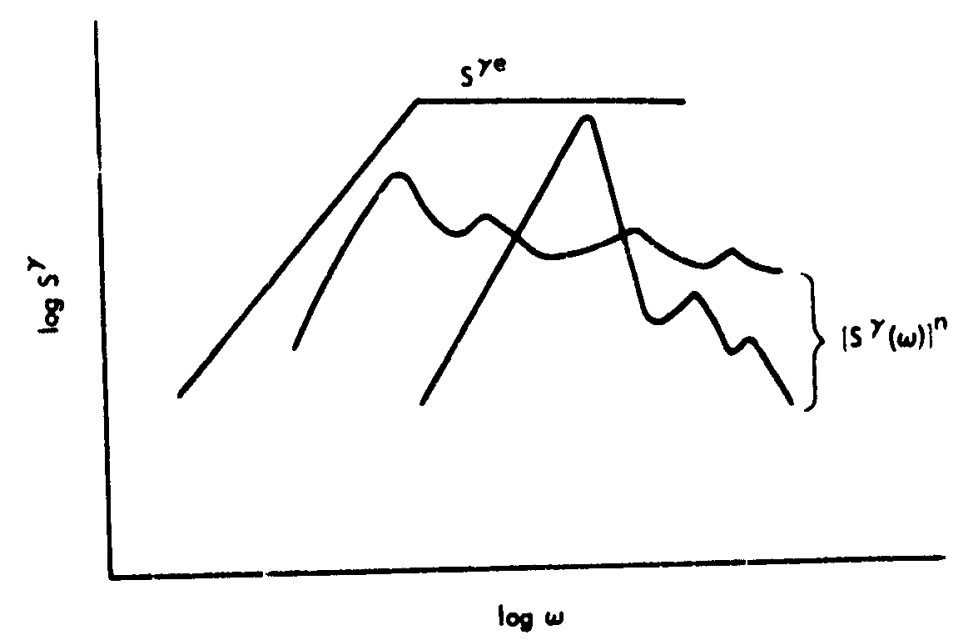

Fig. 2. Shock spectra and envelope 
ensemble of all shock spectra or higher than those peaks depending upon the desired degree of conservatism. This envelope has the advantage of being simple to construct. However, it is very conservative since it has the disadvantage of flattening out all peaks of the shock spectra and therefore ignoring valuable chai acteristics of the source of the base acceleration $\mathrm{ii}_{1}^{\mathrm{O}}(\mathrm{T})$, namely, the peaking effect in terms of frequency. This effect will be reintroduced in the method described.

\section{USE OF THE SHOCK SPECTRA}

The shock spectra envelope $S^{\text {Ye }}$ can be utilized to determine the spacecraft/launch vehicle interface load and also the loads in the eritire spacec $=$ aft. The oscillator (Fig. 1) can represent each cantilevered mode of the spacecraft. Calling $\bar{m}^{i}$ the effective mass of a mode and $\gamma^{i}$ the damping of that mode, then the maximum reaction force on the base of the spacecraft for that mode can be written as:

$$
F_{1}^{0}=\bar{m}^{i} S_{i}^{j e}
$$

where $S_{i}^{\gamma e}$ is the value of the shock spectra envelope corresponding to the damping $\gamma^{i}$ and the natural frequency $\omega^{i}$ of that mode. Note that Eq. (3) relates the loads directly to the shock spectra and with the proper damping, and therefore represents a significant improvement over the traditional method of relating loads to static or sinueoidal acceleration.

However, Eq. (3) can be further improved by noting (1) that the envelope $S^{\gamma e}$ ignores the peaking character of each shock spectrum and (2) that the impedance of the spacecraft is not infinitesimal compared to that of the launch vehicle. The relative impedance between the spacecraft and launch vehicle reduces the interface motion and the reaction on the spacecraft and also shifts the frequency at which the maximum loading occurs.

Therefore, a reduction factor $C \leq 1$ is introduced which depends on the shape of shock spectra peaks as well as the relative impedance, and Eq. (3) is modified as follows:

$$
F_{1}^{R}=m^{1} s_{i}^{Y e} c
$$


The superscript $R$ has been introduced as a reminder that this reaction has been reduced as a function of the relative impedance and peak shape. The object of the next section is to determine the reduction factor $c$.

\section{DETERMINATION OF THE REDUCTION FACTOR}

\section{Equivalent Forcing Function}

To find the reduction facto: $\mathrm{C}$ it is convenient to introduce a hypothetical sinusoidal forcing function $F_{E} e^{j \omega T}$ applied at any convenient point $E$ of the launch vehicle (Fig. 3 ), which for a given frequency w produces an interface motion with the same shock spectra as for the flight data.

An expression of $F_{E}$ proportional to $S^{\text {ye }}$ is sought. Let us assume that $F_{E}$ is applied to the unloaded launch vehicle, i.e., without the spacecraft (Fig. 4).

Using the mobility concept (Ref. 1) for the unloaded launch vehicle, we find that the interface velocity $\left.\dot{u}_{I E}\right\}$ is the mobility $\left\{M_{I E}^{L}\right\}$ at the interface due to a load at point $E$ times the load $F_{E}$ at point $E$.

$$
\left\{\dot{u}_{I E}\right\}=\left\{M_{\text {IE }}^{L}\right\} F_{E}
$$

The mobility $\left\{M_{\text {IE }}^{L}\right\}$ of the launi:h vehicle is the sum of the mobility due to each of its modes $k$. In terms of modal frequercy $\omega^{k}$, riodal

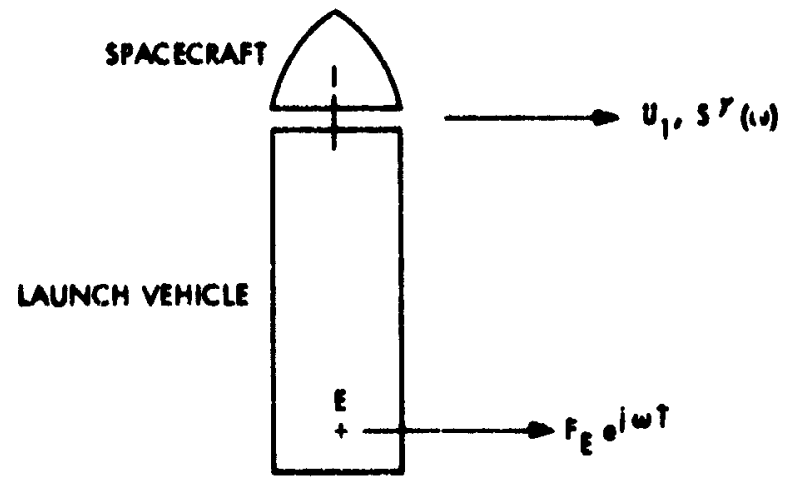

Fig. 3. Composite structure

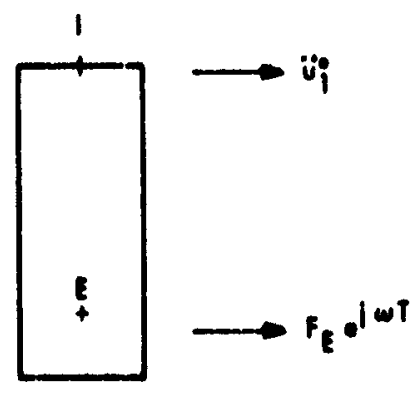

Fig. 4. Unloaded launch vehicle 
mass $\mathrm{m}^{k}$, modal damping $\gamma^{k}$, modal displacements $\phi_{I}^{k}, \phi_{E}^{k}$, and the frequency w we have

$$
M_{I E}^{L}=\sum_{k} \frac{\phi_{I}^{k} \phi_{E}^{k} m^{k} \gamma^{k}(\delta j+1)}{\omega^{k} j}
$$

where

$$
\delta=\left(\frac{\omega}{\omega}-\frac{\omega}{\omega}\right) / \gamma^{k}
$$

Equation (6) exhibits peaks that occur at each resonant frequency of the unloaded launch vehicle. Assuming that the source of the disturbance has a relatively flat frequency distribution, we find that the shock spectra of the unloaded interface accelerations will also have peaks at the same resonant frequencies. The envelope $S^{\gamma e}$ of all shock spectra will be controlled by the amplitude of those peaks. This observation is fundamental for the handling of $s^{\gamma e}$.

Let us now note that in practice, flight measurements are most likely made only for a loaded interface, i.e., for the launch vehicle carrying a payload. However, since the payload has a small mass compared to the launch vehicle, the presence of this payload does not appreciably change the amplitude of the peaks of the unloaded launch vehicle interface acceleration shock spectra (Appendix A), although the frequencies of those peaks are somewhat shified. In addition, secondary peaks will occur near the cantilevered spacecraft frequencies, but these peaks will be lower than those due to the launch vehicle and hence will not control the shock spectra envelope sye.

It is therefore postulated that the shock spectra envelope $s^{\gamma e}$ is controlled only by the amplitude of the launch vehicle peaks and is unaffected by the presence of the spacecraft; 1.0. , the shock spectra envelope can be determined from flights with a loaded interface. Note that the postulate is not valid if the data comes from a flight where a launch vehicle resonant 
frequency is tuned to a spacecraft resonant frequency. In this case, the controlling launch vehicle peak will have a lower amplitude than for an untuned case (Appendix A). However, this tuned case is unlikely to occur for all spacecraft modes of all prior flights; the onvelope will be determined by the untuned case or by other flights without tuning.

It was noted earlier in Section II-B-2 that the envelope $\mathrm{s}^{\text {ye }}$ flattens out the peaks of the real shock spectra $\left[S^{\gamma}(\omega)\right]^{n}$. In order to reintroduce the peaking characteristic of the real shock spectra, a hypothetical curve $S(\gamma, \alpha)$ enveloping any $\left[s^{\gamma}(\omega)\right]^{n}$ is defineu as $S(\gamma, \alpha)=s^{\gamma e} / \sqrt{\delta^{2} \alpha+1}$ for $\delta^{2} \alpha \leq 1$ (Fig. 5). This enveloping curve has the amplitude of the peak at the level $S^{\gamma e}$ of the simple envelope but allows the peak to shift in frequency along $s^{\gamma e}$. The coefficient $\alpha$ controls the sharpness of the enveloping curve and is chosen such that all peaks of each available shock spectra data are enveloped. The limitation of $\delta^{2} \alpha \leq 1$ corresponds to the half-power point cutoff to account for close peaks and background noise that would otherwise create problems in implementation.

The amplitude $F_{E}$ of the equivalent forcing functions will now be determined. To relate $F_{E}$ to the flight acceleration shock spectra envelope we attach to the unloaded interface an infinitesimal oscillator of mass $\bar{m}^{i}$, natural frequency $\omega^{i}$, and damping $\gamma^{i}$ (Fig. 6).

The maximum amplitude of the velocity response $\dot{u}_{i}$ of the mass of the oscillator due to $F_{E}$ occurs at $\omega=\omega^{i}$ and is given by

$$
\dot{u}_{1}=\dot{u}_{1}^{O} / j \gamma^{i}=M_{1 E}^{L} F_{E} / j \gamma^{i}
$$

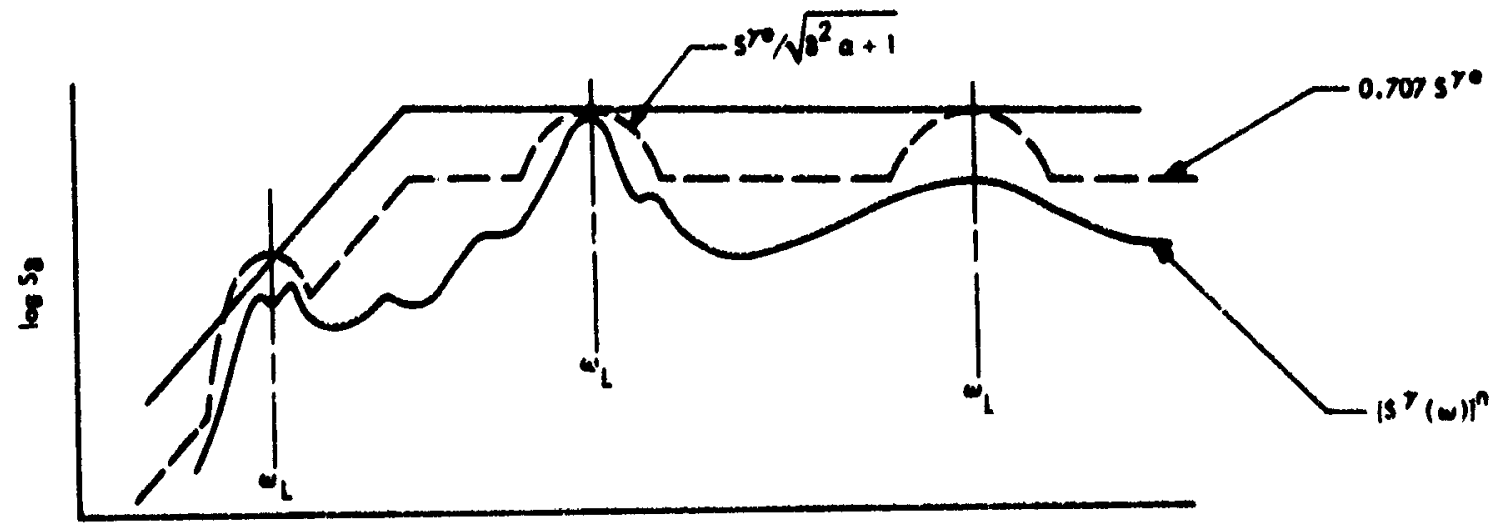

100

Fig. 5. Hypothetical curve enveloping peaks of shock opectra 


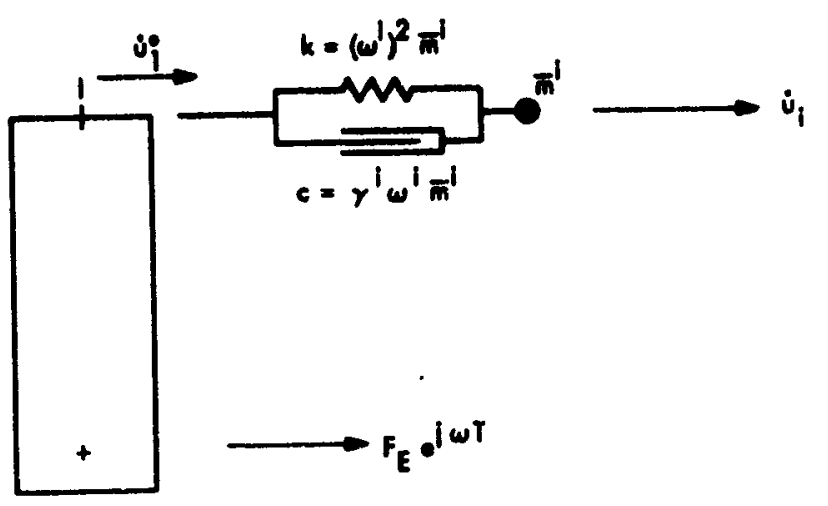

Fig. 6. Infinitesimal oscillator on unloaded interface

The amplitude of the equivalent force $F_{E}$ is obtained by equating $i_{i}$ with the shoci spectra envelope modified by the assumed shape of a shock spectra peak. We have

$$
\dot{u}_{i} \leq s^{\gamma e} / j \omega \sqrt{\delta^{2} \alpha+1}
$$

Since this envelope depends only on the amplitude of the peaks, one can consider an interface motion due to only one launch vehicle mode at a time. Then combining Eqs. (7) and (8) the equivalent force $F_{E}$ is

$$
F_{E} \leq \frac{\gamma^{i}}{M^{k}} s^{\gamma e} / \omega \sqrt{\delta^{2} \alpha+1}
$$

where $M^{k}$ is the mobility correeponding to one launch vehicle mode in Eq. (6), in which the arbitrary location of point $\mathbf{E}$ has been taken at the interface 1 for convenience $\left(\phi_{\mathbf{E}}=\phi_{1}\right)$. The equivalent force $F_{\mathbf{E}}$ is an external force at the interface 1 ,

$$
M^{k} \equiv \frac{\phi_{1}^{2}}{m^{k} y^{k} \omega^{k}(6 j+1)}
$$




\section{Interface Reaction}

When the launch vehicle is loaded by the spacecraft (Fig. 7), a reaction force $\left\{F_{I}\right\}$ is induced by the spacesraft on the launch vehicle interface.

For the launch vehicle the interface velocity $\left\{\dot{u}_{i}\right\}$ is the mobility $\left\{M_{I E}^{L}\right\}$ at the interface $I$ due to a force at $E$ times the force $F_{E}$ at $E$, plus the mobility $\left[M_{I I}^{L}\right]$ at the interface due to a force at the interface, times the reaction force $\left\{F_{I}\right\}$ at the interface $I$ :

$$
\left\{\dot{u}_{I}\right\}=\left[\begin{array}{l:l}
M_{I E}^{L} & M_{I I}^{L}
\end{array}\right]\left\{\begin{array}{c}
F_{E} \\
\hdashline F_{I}
\end{array}\right\}
$$

For the spacecraft the interface velocity is the mobility $\left[M_{I I}^{S}\right]$ at the interface due to the force at the interface, times the reaction $\left\{-F_{I}\right\}$ on the spacecraft:

$$
\left\{\dot{u}_{I}\right\}=\left[M_{I I}^{S}\right]\left\{-F_{I}\right\}
$$

Combining Eqs. (11) and (12):

$$
\left\{M_{I E}^{L}\right\} F_{E}=-\left[M_{I I}^{L}+M_{I I}^{S}\right]\left\{F_{I}\right\}
$$

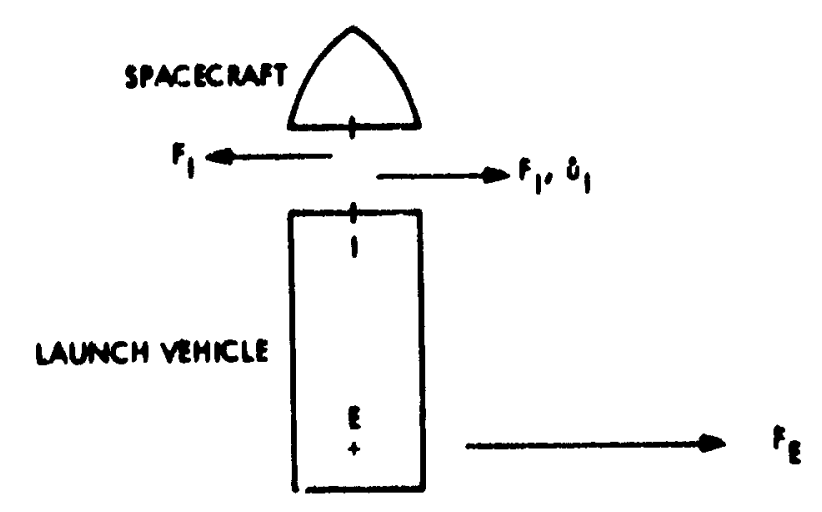

Fif: 7. Launch vehicle/spacecrafl composite otructure 
Rearranging Eq. (13) and using the superscript $R$ as a reminder that $\left\{F_{I}\right\}<\left\{F_{I}^{O}\right\}$,

$$
\left\{F_{I}^{R}\right\}=-\left[M_{I I}^{L}+M_{I I}^{S}\right]^{-1}\left\{M_{I E}^{L}\right\} F_{E}
$$

In Eq. (14) the mobilities are three-dimensional and contain all the modes of the spacecraft and the launch vehicle. However, the essence of the method leading to the reduction factor $C$ can be presented at the singledegree-of-freedom level. Thus, the development of the method will utilize single-degree-of-freedom models, one for the spacecraft and one for the launch vehicle, with the data point at the interface and along the line of motion (Fig. 8). This special case will then be generalized.

The modal mobilities $M^{k}$ for the launch vehicle, Eq. (10), and $M^{i}$ for the spacecraft will be substituted for $M_{I I}^{L}$ and $M_{I I}^{S}$ respectively, in Eq. (14). The modal mobility $M^{i}$ is the sum of the mobility of the effective mass $\bar{m}^{i}$ and the mobility of the modal spring damper system connecting $\frac{i}{m}$ to the interface (Fig. 9).

$$
M^{i}=\frac{1}{j \bar{m}^{i} \omega}+\frac{1}{c^{i}+k^{i} / \omega j}
$$

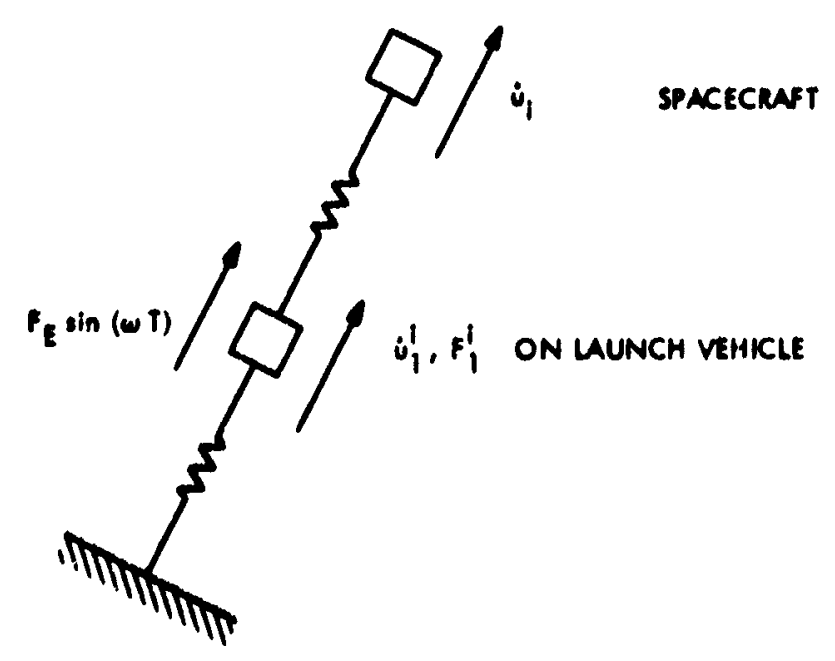

Fig. 8. Modal model of composite structure 


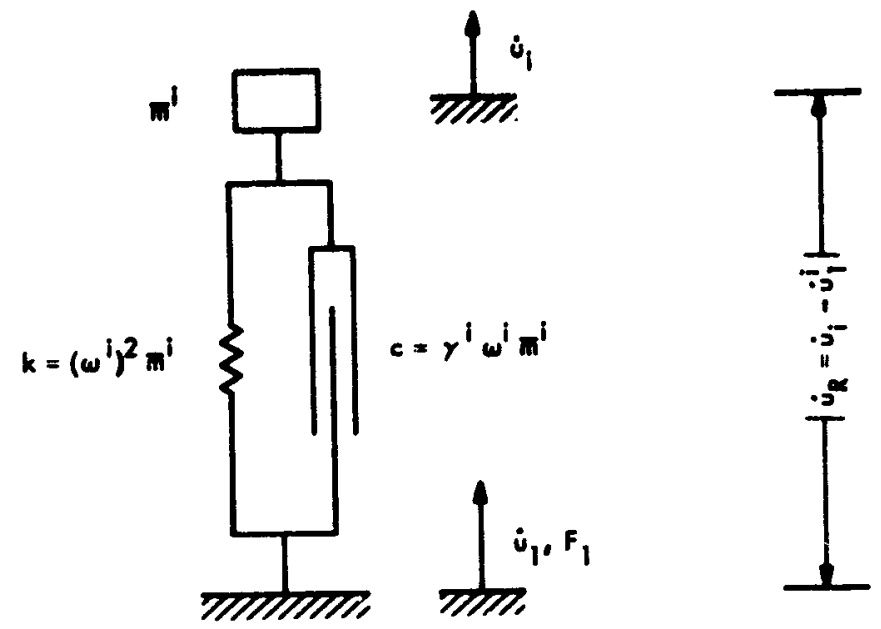

Fig. 9. Relative velocities for cantilevered modes

The effective mass $\overline{\mathrm{m}}^{\mathrm{i}}$ is determined as the ratio of rigid/elastic mass coupling squared to the modal mass (Ref. 1).

$$
\bar{m}^{i}=\frac{1}{m^{i}}\left\{m_{I i}\right\}^{T}\left\{m_{I i}\right\} \quad I=\text { translation only }
$$

Expressing the damping and siiffness of Eq. (15) in terms of modal frequency $\omega^{i}$, damping ratio $\gamma^{i}$, and effective mass $\overline{\mathrm{m}}^{i}$, we obtain:

$$
M^{i}=\frac{1}{\omega^{i} m^{i}}\left(\frac{1}{j \frac{\omega}{\omega^{i}}}+\frac{1}{\gamma^{i}-\frac{\omega^{i}}{\omega} j}\right)
$$

Introducing $\varepsilon=\left(\omega / \omega^{i}-\omega^{i} / \omega\right) / \gamma^{i}$ in Eq. (17) and neglecting $\gamma^{i}\left(\omega / \omega^{i}\right)$ as compared to unity we finally have

$$
M^{1} \times \frac{(\varepsilon j+1)^{\gamma^{1}}}{\omega^{1} \bar{m}^{1}}
$$


In addition, for single-degree-of-freedom models, Eq. (14) reduces to

$$
F_{1}^{R}=-\frac{M^{k} F_{E}}{M^{i}+M^{k}}
$$

Substituting Eqs. (9), (10), and (18) into Eq. (19) and considering the absolute value, the following expression is derived for the reaction force:

$$
F_{i}^{R}=\bar{m}^{i} s^{\gamma e} \mathscr{B}
$$

where

$$
\mathscr{C}=\frac{\omega^{i}}{\omega} \sqrt{\frac{\delta^{2}+1}{\left[(\theta+1-\delta \varepsilon)^{2}+(\delta+\varepsilon)^{2}\right]\left(\delta^{2} \alpha+1\right)}}
$$

with

$$
\begin{aligned}
& \delta=\frac{\left(\frac{\omega}{\omega^{k}}-\frac{\omega^{k}}{\omega}\right)}{\gamma^{k}} \\
& \varepsilon=\frac{\left(\frac{\omega}{\omega^{i}}-\frac{\omega^{i}}{\omega}\right)}{\gamma^{i}} \\
& \theta=\frac{\omega^{i}}{\omega^{k}} \frac{\bar{m}^{i} \phi_{1}^{2}}{m^{k}} \frac{1}{\gamma^{i} \gamma^{k}}
\end{aligned}
$$

For structural design, we are interested in the maximum value that the reaction $F_{1}^{R}$ can reach. This reaction depends upon two parameters, $b$ and $\varepsilon$, for given $y^{i}, y^{k}, \theta$, and $\alpha$. The two parameters $\delta$ and $\varepsilon$ measure the proximity of the two resonant frequencies $w^{1}$ of the spacecraft and $\omega_{k}$ of the launch vehtcle and the dummy frequency $w$. 
A common approach for lighter-weight design is to avoid tuning between the two resonant frequencies $\omega^{i}$ and $\omega^{k}$. However designing for wellseparated resonant frequencies requires costly, accurate structural analysis and is difficult to achieve because of unpredictable variation in fabrication tolerances. The method proposed here is to provide for the tuned condition even if tuning will not exist in practice. The method, although not optimum for weight, is cost-effective. It also increases reliability since only simplified structural analysis is required and the design loads a re for inadvertent tuning that may occur from resonant frequency shifts due to fabrication tolerances. Heuristically, it is expected that the maximum of the reaction $F_{1}^{R}$ occurs for $\omega^{i}=\omega^{k}=\omega$. However, close examination of Eq. (22) shows that the maximum of $F_{1}^{R}$ occurs for a condition somewhat different from that of tuning. The maximum value $C$ of the coefficient $\mathscr{B}$ in terms of the two independent variables $\delta$ and $\varepsilon$ (correspondingly $\omega^{i} / \omega$ and $\omega^{k} / \omega$ ) has been determined numerically for convenience. Therefore, Eq. (20) becomes

$$
\begin{aligned}
F_{1}^{R} & \leq \bar{m}^{i} s^{\gamma e} C \\
C & =C\left(\gamma^{i}, \gamma^{k}, \theta^{l}, \alpha\right) \\
\theta^{1} & =\frac{\omega^{k}}{\omega^{i}} \theta
\end{aligned}
$$

The values of $C$ as a function of $\gamma^{i}, \gamma^{k}$, and $\theta^{l}$ are shown in Fig. 10 for values of a given in Fig. 11 .

\section{Typical Values of $\alpha$}

Typical values of the parameter $\alpha$ defining shock spectra peak shapes for the Titan IIIC booster are given in Fig. 11. The accelerations from which the shock spectra were obtained are at the top of the Centaur stub adapter. There is a great deal of scatter in the data, but a trend toward sherper peaks with iower damping is evident. A curve whose slope is approsimately that of the trend is also shown. The analytic expression for that curve is 


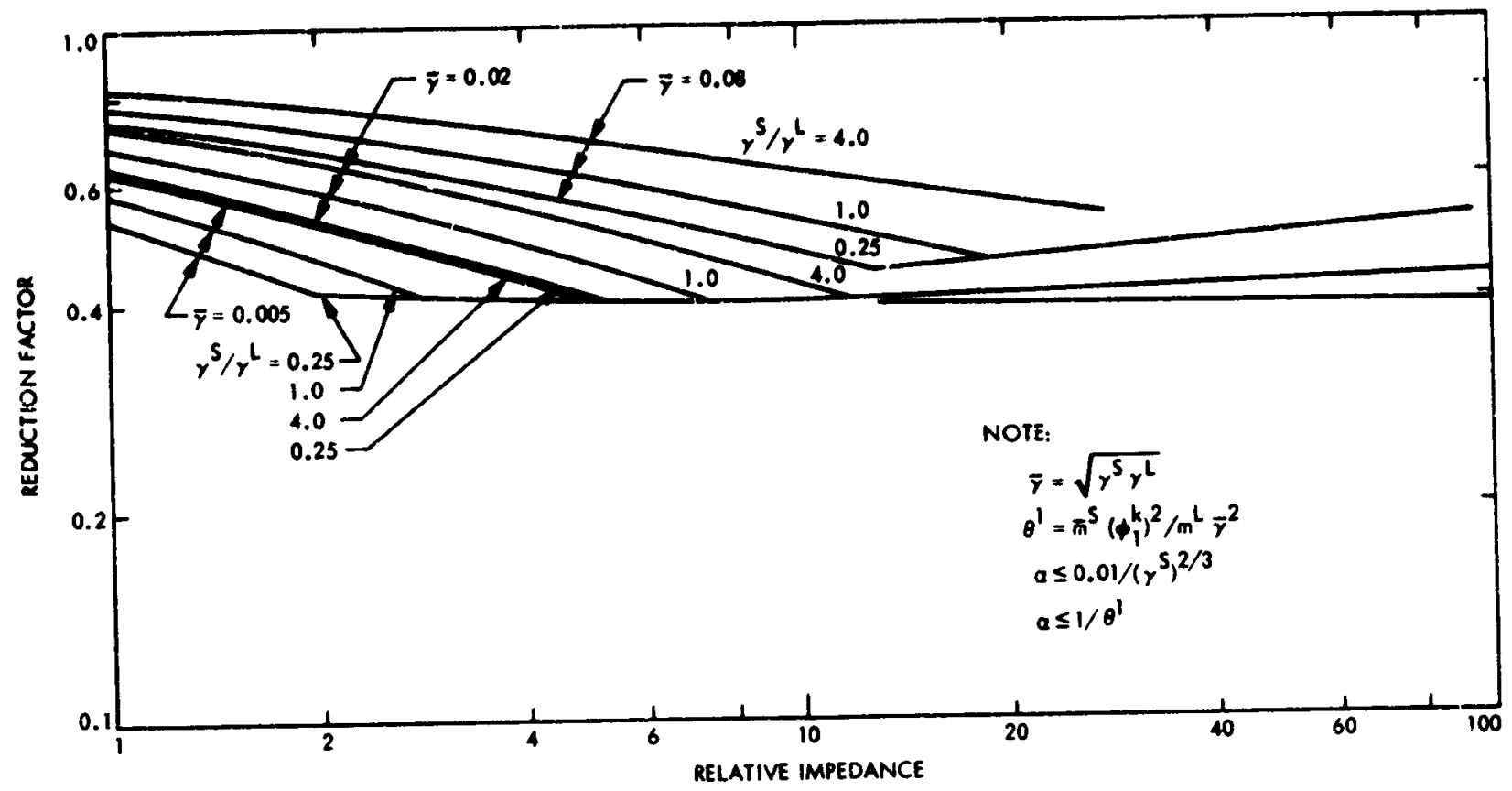

Fig. 10. Response reduction due to relative impedance

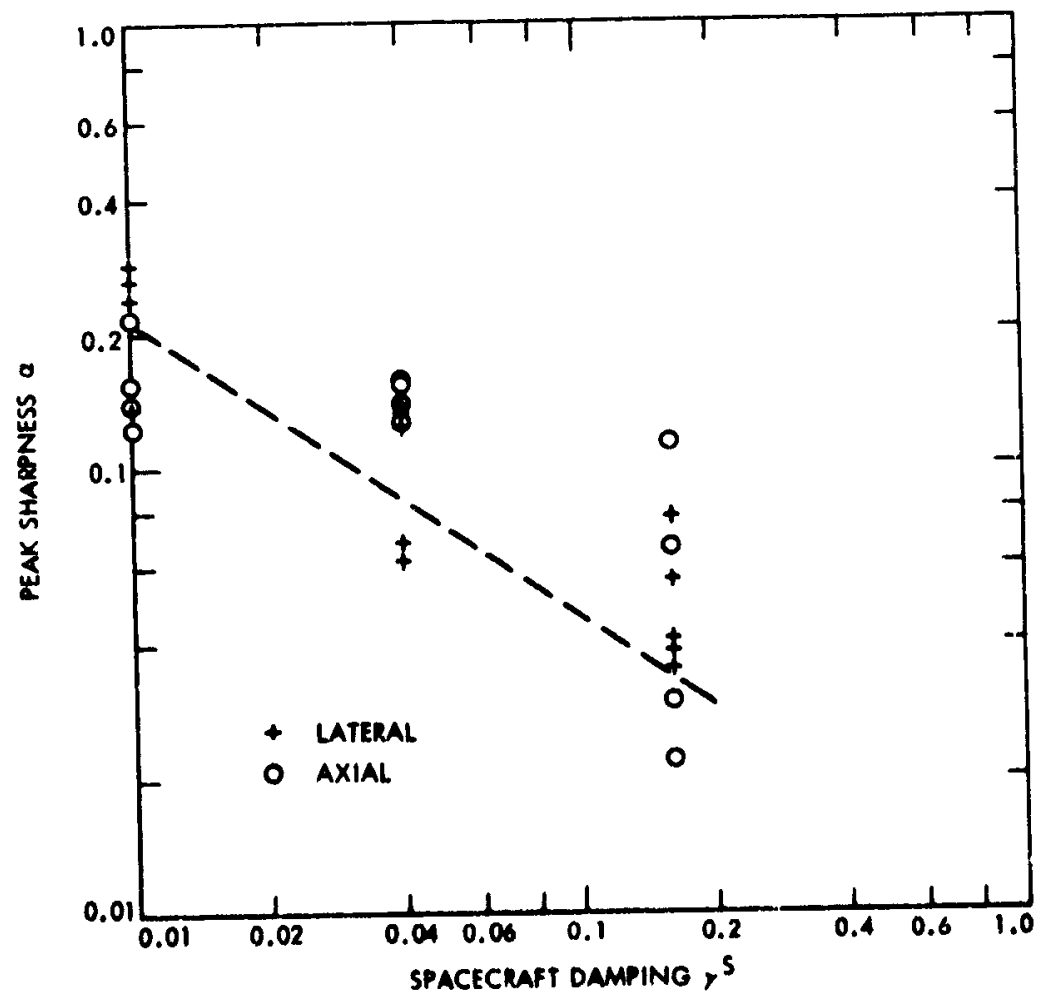

Fig. 11. Shock spectra peak sharpness for top of Centaur stub adaptor 


$$
\alpha=\frac{0.01}{\left(\gamma^{i}\right)^{2 / 3}}
$$

No atternpt has been made to find a trend with respect to frequency.

Values of $\alpha$ greater than $1 / \delta^{2}$ will not be utilized as indicated in Section II-D-1.

\section{Typical Values of $\mathrm{C}$}

The approximation to $\alpha$ is used to evaluate values of $C$ which can be used for payloads supported on the Titan IIIC booster. The values of $\mathrm{C}$ shown in Fig. 10 indicate that $C$ is more sensitive to the geometric mean $\bar{\gamma}=\sqrt{\gamma^{i} \gamma^{k}}$ of spacecraft and launch vehicle damping than to either one separately. The reduction effect is greater for light damping, but the shock spectra is expected to be higher for light damping. Thus, the two effects of damping oppose each other, and the reaction is not overly sensitive to damping.

\section{Typical Shock Spectra Envelopes}

Shock spectra envelopes for axial and lateral acceleration at the top of the rtub adaptor of the Titan IIIC are shown in Fig. 12. The se envelopes are based on simulated flights using engine chamber pressures to generate forcing functions and both Viking and Helios spacecraft models.

\section{Damping}

If the spacecraft damping is not known accurately, the reaction can still be bounded by evaluating the reaction using both a low and a high estimate of the damping in combination with the corresponding shock spectra.

\section{E. GENERALIZATION}

\section{Modal Reaction Force}

The derivation of the reaction force $F_{1}^{R}$ in Section II-D-2 was done for a single-degree-of-freedom uscillator along its line of motion. The 


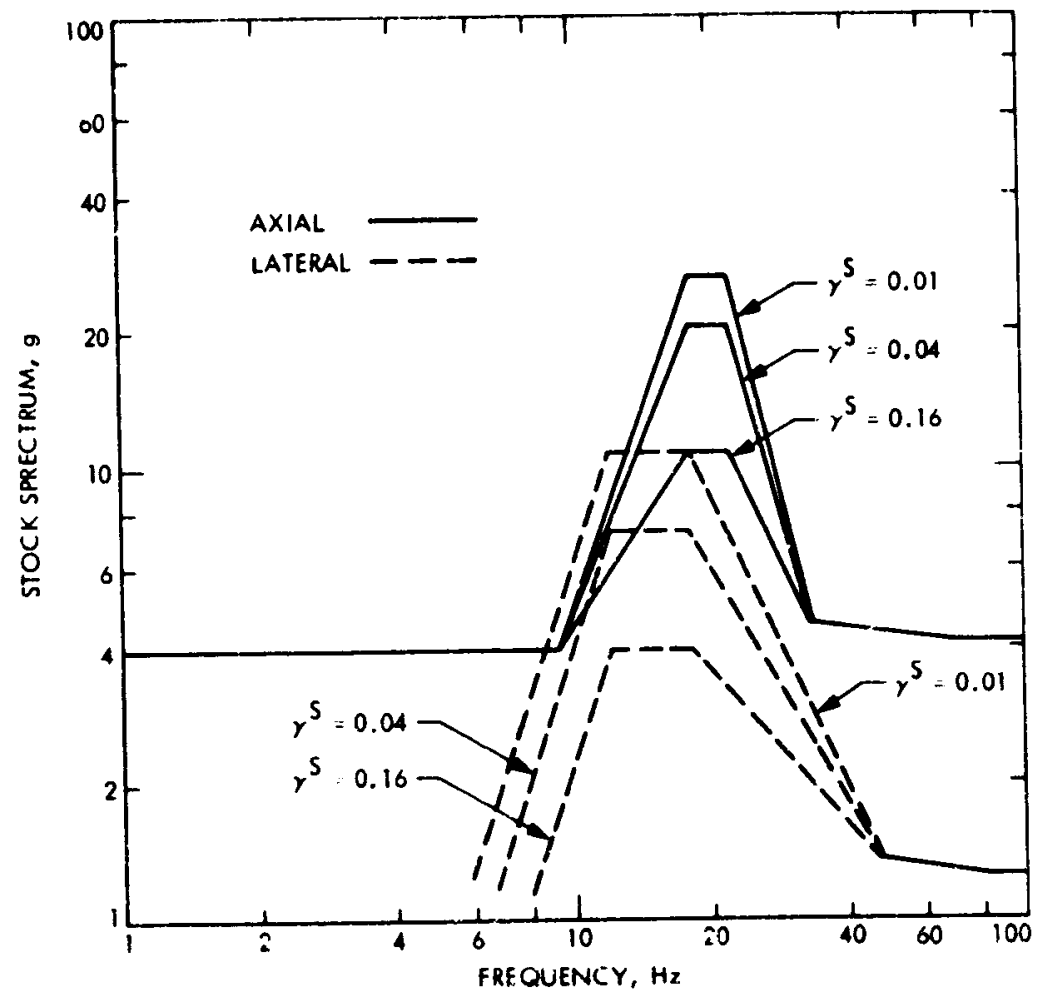

Fig. 12. Shock spectra envelopes at top of Titan III-C

derivation will now be generalized to a multicimensional interface I to determine the multidimensional reaction force $\left\{F_{I}\right\}$ at this interface.

Call $\left\{\bar{m}_{I i}\right\}$ the mass coupling between a spacecraft cantilever mode of effective mass $\bar{m}_{i}$ and the unit rigid body interface motions; then the equivalent interface motion $u_{1}^{i}$ (Fig. 8) along a single.degree-of-freedom representation of the mode $i$ is $\left.(1 / \overline{\mathrm{m}} \mathrm{i})\right|_{\mathrm{m}}\left|{ }_{\mathrm{I}}\right| \mathrm{T}$ (Ref. 2), times the real motion $\left\{u_{1}\right\}$ at the interface $I$.

$$
u_{1}^{i}=-\frac{1}{\bar{m}^{i}}\left|\bar{m}_{I i}\right| T\left\{u_{I}\right\}
$$

The reaction vector $\left\{F_{I}^{i}\right\}$ at the $I$ interface is $\left.\left(1 / \bar{m}^{i}\right) \mid \bar{m}_{1 i}\right\}_{\text {times }} F_{1}^{R}$ previously calculated, along the line of motion of the single-degree-of-freedom system

$$
\left\{F_{I}^{i}\right\}=\frac{1}{\bar{m}^{i}}\left\{\bar{m}_{I i}\right\} F_{1}^{R}
$$


The motion $\left\{u_{I}\right\}$ at the interface $I$ is the mode shape $\left\{\phi_{I}^{k}\right\}$ of that interface for mode $k$ times the modal motion $U^{k}$ of the launch vehicle

$$
\left\{u_{I}^{k}\right\}=\left\{\phi_{I}^{k}\right\} U^{k}
$$

The motion at the data point $u_{D}$ is the mode shape $\phi_{D}^{k}$ evaluated at the data point times the modal motion of the launch vehicle $\mathrm{U}^{\mathrm{k}}$ :

$$
u_{D}^{k}=\phi_{D}^{k} U^{k}
$$

From Eqs. (26) and (27) the motion at the interface is

$$
\left\{u_{I}^{k}\right\}=\frac{u_{D}^{k}}{\phi_{D}^{k}}\left\{\phi_{1}^{k}\right\}
$$

$T$ : shock spectra peaks $S_{\bar{I}}^{\gamma^{k}}$ and envelopes can be scaled by the same factors since they are due to launch vehicle modes,

$$
\left\{S_{I}^{\gamma k}\right\}=\frac{S_{D}^{\gamma}}{\phi_{D}^{k}}\left\{\phi_{I}^{k}\right\}
$$

This allows us to use flight data that has been recurded at points other than the interface. Analogous to Eq. (24),

$$
s_{1}^{\gamma^{i k}}=\frac{1}{\bar{m}^{i}}\left\{\bar{m}_{I i}\right\}^{T}\left\{s_{I}^{\gamma k}\right\}
$$

Ccmbining Eqs, (23), (25), (29), and (30),

$$
\left\{F_{I}^{i k}\right\}=\frac{1}{m^{i}}\left\{m_{I i}\right\} m^{i} \frac{1}{m^{i}}\left\{m_{I i}\right\}^{T} \frac{s_{D}^{Y e}}{\phi_{D}^{k}}\left\{\phi_{1}^{k}\right\} c^{i k}
$$


which simplifies to

$$
\left\{F_{I}^{i k}\right\}=\frac{S_{D}^{\gamma e} C^{i k}}{m^{i} \phi_{D}^{k}}\left\{m_{I i}\right\}\left\{m_{I i}\right\}^{T}\left\{\phi_{I}^{k}\right\}
$$

where the bar has been omitted for $m_{i}$ and $\left\{m_{I i}\right\}$ since the triplet

$$
\frac{1}{m_{i}}\left\{m_{I i}\right\}\left\{m_{I i}\right\}^{T}
$$

is independent of modal normalization. The normalization for an effective mass $\bar{m}_{i}$, if used, should be applied to both the mass coupling $\left\{\mathrm{m}_{\mathrm{Ii}}\right\}$ and the mode shapes $\left\{\phi^{i}\right\}$ of the spacecraft. The square of the factor is applied to $\mathrm{m}^{i}$ so its effect on the reaction and later on the displacement vector cancels. This is expected since the method of normalization cannot affect the results but is a useful aid in interpreting the model.

For parallelism with Eq. (23) the following definitions are made:

$$
\begin{aligned}
& {\left[\bar{m}_{I I}^{i}\right] \equiv \frac{1}{m^{i}}\left\{m_{I i} \mid m_{I i}\right\}^{T}} \\
& \left\{s_{I}^{y k e}\right\}=\frac{s_{D}^{\gamma e}}{\phi_{D}^{k}}\left\{\phi_{I}^{k}\right\}
\end{aligned}
$$

Substituting Eqs. (33) and (34) into Eq. (32), we obtain

$$
\left\{F_{I}^{i k}\right\}=C^{i k}\left[m_{I I}^{i}\right]\left\{s_{I}^{\gamma k e}\right\}
$$

Note that $\left[\mathrm{m}_{11}^{i}\right]$ and $\left\{F_{1}^{i k}\right\}$ are a matrix and a vector with order equal to the number of degrees of freedom at the interface. 
2. Evaluation of the Reduction Factor $C^{i k}$

The reduced reaction $F_{I}^{i k}$ has the same form for the single-degree-offreedom models of Section II-D-2. To evaluate $C^{i k}$, curves of Fig. 10 are used for which the coefficient $\theta^{l}$ becomes $\theta^{i k}$ as follows. Froin Eqs. (24) and (26) we have:

$$
u_{1}^{i k}=\frac{1}{\bar{m}^{i}}\left\{\bar{m}_{I i}\right\}^{T}\left\{\phi_{I}^{k}\right\} U^{k}
$$

The part preceding $U^{k}$ in Eq. (36) is the mode shape $\phi_{1}^{i k}$ that replaces $\phi_{1}$ of Section II-D-2.

$$
\phi_{1}^{i k} \equiv \frac{1}{\overline{\mathrm{m}}^{\mathrm{i}}}\left\{\overline{\mathrm{m}}_{\mathrm{Ii}}\right\}^{\mathrm{T}}\left\{\phi_{\mathrm{I}}^{\mathrm{k}}\right\}
$$

Substituting Eq. (37) in the definition of $\theta$, we obtain

$$
\theta^{i k} \equiv \frac{\omega_{i}}{\omega_{k}} \frac{\bar{m}^{i} \frac{1}{m^{i}}\left\{\bar{m}_{I i}\right\}^{T}\left\{\phi_{I}^{k}\right\} \frac{1}{m^{i}}\left\{\bar{m}_{I i}\right\}^{T}\left\{\phi_{I}^{k}\right\}}{m^{k}} \frac{1}{\gamma^{i} \gamma^{k}}
$$

which simplifies to

$$
\theta^{i k}=\frac{\omega^{i}}{\omega^{k}} \frac{\left\{m_{I 1}\right\}^{T}\left\{\phi_{I}^{k}\right\}\left\{m_{I 1}\right\}^{T}\left\{\phi_{I}^{k}\right\}}{m^{i} m^{k} \gamma^{i} \gamma^{k}}
$$

Note again that the renormalization of the modes to obtain the effective mass $m_{1}$ is not required in Eq. (39) as was the case for Eq. (37).

\section{Modal Member Loade}

Member loads $\left\{p^{1}\right\}$ due to each mode are obtained by premultiplying the displacement vector $\left\{u^{1}\right\}$ of the spacecraft by the stress coefficient matrix [sC] of the member. 


$$
\left\{p^{i}\right\}=[\operatorname{sc}]\left\{u^{i}\right\}
$$

The displacement vector $\left\{u^{i}\right\}$ is obtained by multiplying the spacecraft mode $\left\{\Phi^{i}\right\}$ normalized for equivalent effective mass by the relative motion $u_{R}=u_{i}^{k}-u_{1}$ (Fig. 9) of the effective mass representation of the spacecraft mode.

A conservative estimate of member loads for spacecraft mode $i$ and launch vehicle mode $k$ is obtained by replacing $u_{R}$ by $i_{i}^{k *} \equiv S_{D}^{\gamma e}-\ddot{u}_{I}^{\max }$. The shock spectra at the data point is che sum of the data point acceleration $\mathrm{u}_{\mathrm{D}}$ and the relative acceleration $\mathrm{i}_{\mathrm{R}}$ of the oscillator. The contribution of each is not known since such information is lost in the total acceleration shock spectra processing. However, since the interface base acceleration will be used without reduction while the modal acceleration will be multiplied by a factor less than 1.0, overestimates of the base acceleration contribution are conservative (see Appendix B). The maximum value of the data point acceleration $\ddot{u}_{D}$ as measured by the tail of the shock spectra at frequencies greater than those of interest will be used as the estimate of the interface base acceleration $\mathfrak{i}_{i}$ in the same direction. It is also conservative to assume that the rigid body and modal acceleration are in phase. Therefore, it is conservative to estimate the relative acceleration as the data point shock spectra less any interface base acceleration of significance. The relative motion vector $\left\{u^{i}\right\}$ is then estimated as:

$$
\left\{u^{i}\right\} \cong\left\{\bar{\phi}^{i}\right\} u_{i}^{k *}
$$

The tutal motion $u_{i}^{k *}$ in Eq. (41) is obtained from the acceleration estimate $u_{i}^{k *}$ of the effective mass $\bar{m}_{i}$ of the spacecraft and the reaction force

$$
u_{1}^{k *}=\frac{\ddot{u}_{i}^{k *}}{\omega^{2}}=\frac{F_{1}^{R}}{\bar{m}_{1} \omega^{2}}
$$


Using Eqs. (25), (32), and (42), we have, at $\omega=\omega^{i}$,

$$
u_{i}^{k} \leq \frac{c^{i k}\left(s_{D}^{y e}-i_{I}^{\max }\right)}{\left(\omega^{i}\right)^{2} \phi_{D}^{k} \bar{m}^{i}}\left\{\bar{m}_{I i}\right\}^{T}\left\{\phi_{I}^{k}\right\}
$$

Combining Eqs. (40), (41) and (43) we obtain the member loads

$$
\left\{p^{i}\right\} \leq \frac{c^{i k}\left(s_{D}^{\gamma e}-\ddot{u}_{I}^{\max }\right)}{\left(\omega^{i}\right)^{2} \phi_{D}^{k}}[S C] \frac{1}{m_{i}}\left\{\phi^{i}\right\}\left\{m_{I i}\right\}^{T}\left\{\phi_{I}^{k}\right\}
$$

where again the bar has been omitted.

\section{Total Loads}

In the previous sections only the modal loads for one pair of modes are calculated at a time. Consideration will now be given to the combinations of the modal loads to obtain a bound of the total loads. This combination presents a difficulty for the present method. When excited, all the modes are not responding "in phase," so load maxima are not occurring simultineously for ali modes. However, since the modal loads developed in the previous sections are bounds, the phase information is not available.

A bound of the total load can be readily calculated by assuming that at some point in the time history all the modal loado in a given direction will add. That is,

$$
F_{I}^{R} \leq \sum_{I}\left|F_{I}^{i k}\right| \quad \text { in one direction }
$$

and

$$
p \leq \sum_{1}\left|p^{i}\right| \text { in one direction }
$$


This bound is overly conservative unless only one pair of modes is predominant over all others.

If two or more pairs give similar modal loads, their relative occurrence should be examine!. This would be particularly true if the frequencies of the pairings are widely separated. Since the excitation is transient in nature, one maxima is most likely to occur well before the other.

The conservatism built into Eqs. (45) and (46) can be greatly reduced by introducing some elementary statistics in the formulation for the combination of modal loads. In Eqs. (45) and (46) it is assumed that all modes of the spacecraft are paired and tuned simultaneously with a mode of the launch vehicle. This situation, although theoretically possible, is actually quite unlikely in practice since only a few, if any, spacecraft modes will be tuned to the launch vehicle modes. Consequently, one can treat all the bounds of the modal loads as statistical quantities and introduce the root sum square (rss) technique to evaluate the loads as follows:

$$
F_{I}^{R} \leq \sqrt{\sum_{i}\left(F_{I}^{i k}\right)^{2}} \text { with high reliability }
$$

and

$$
p \leq \sqrt{\sum_{i}\left(p^{i}\right)^{2}} \text { with high reliability }
$$

An alternative method to subtracting base motion from total acceleration shock spectra would be to generate relative acceleration shock apectra directly and include rigid body acceleratione nd the associated quasistatic otresses in the RSS oummation. This has not been done, since only a total motion shock opectra evaluation program was on hand.

In the problem used as an example, a spacecraft supported on a launch vehicle, the only oignificant static acceleration 1 s along the vehicle axis, and the data point in that direction 1s elther at the interface or has almost the 
same modal displacement in all modes of interest. For such a problem the method described has the advantage over the alternate method that greater assurance of bounding is possible with negligible penalty.

Let us now make some additional remarks concerning each of the modal loads. Equations (35) and (40) give the desired interface reaction and member loads for mode $i$ of the spacecraft when the motion at the interface is predominantly due to mode $k$ of the launch vehicle. Use of the launch vehicle mode which is the most hostile is conservative. If more than one $S_{D}^{y e}$ is suitable to use, the one resulting in the lowest reaction should be used, as all are bounds.

The lumped mass model of each normal mode represents the rigid body inertial properties as well as the elastic properties. Quasistatic acceleration is represented even for cutoff transients if the quasistatic boundary conditions are included in the derivation of the shock spectra. However, only the dynamic portion of the response is subject to reduction by the factor $\mathrm{C}^{i k}$. The appropriate quasistatic acceleration should be subtracted from the shock spectra before it is used. Also the effect of the quasistatic load must be added unreduced to the summation of the contribution of each mode.

\section{REFERENCES}

1. "Mechanical Impedance Methods for Mechanical Vibrations, " colloquium edited by R. Plunkett, presented at the ASME Annual Meeting, New York, Dec. 2, 1958.

2. Bamford, R. M., Wada, B. K., and Gayman, W. H. , Equivalent Spring Mass Syutem for Normal Modes, Technical Memorandum 33-380, Jet Propuloion Laboratory, Pasadena, Calif. , February 15, 1971 . 


\section{APPENDIX A}

\section{VARIATION OF INTERFACE ACCELERATION IN TERMS OF SPACECRAFT/LAUNCH VEHICLE INTERACTION}

The effect of prior spacecraft on vehicles from which acceleration data was taken is now inspected. Because of the assumption made in Section II-D-1, the amplitude of the effective forcing function at the launch vehicle resonance without a spacecraft is the same as at the composite resonance of a launch vehicle with a spacecraft attached. The ratio $R$ of maximum motions at the interface unloaded and loaded is the ratio of unloaded to loaded maximum mobilities

$$
\Re=\frac{\left(\dot{u}_{1}^{k}\right)^{\max }}{\left(\dot{u}_{1}^{i k}\right)^{\max }}=\frac{\left(M_{11}^{k}\right)^{\max }}{\left(M_{11}^{k i}\right)^{\max }}
$$

The impedance of the loaded vehicle is the sum of the impedance of the spacecraft and the launch vehicle

$$
\frac{1}{M_{11}^{i k}}=\frac{1}{M_{11}^{i}}+\frac{1}{M_{11}^{k}}
$$

The mohility of the unloaded vehicle is (see Section II-D-1)

$$
M_{11}^{k}=\frac{\left(\phi_{1}^{k}\right)^{2}}{\omega^{k} m^{k} y^{k}(\delta j+1)}
$$

The unloaded launch vehicle has a maximum mobility when its imaginary part is zero; i.e., $s=0$.

$$
\left(M_{11}^{k}\right)^{\max }=\frac{\left(b_{1}^{k}\right)^{2}}{\omega^{k} m^{k} y^{k}}
$$


The mobility of the spacecraft is (see Section II-D-1)

$$
M_{11}^{i}=\frac{y^{i}}{\omega^{i} \bar{m}^{i}}(\varepsilon j+1)
$$

Therefore, Eq. (A-2) becomes, after reduction

$$
\frac{1}{M_{11}^{i k}}=\frac{1}{\left(M_{11}^{k}\right)^{\max }}\left\{1+\frac{\theta}{\varepsilon^{2}+1}+\frac{\left[\left(1+\varepsilon^{2}\right) \delta-\theta \varepsilon\right] j}{\varepsilon^{2}+1}\right\}
$$

The loaded launch vehicle mobility $M_{11}^{\text {ik }}$ is a minimum for a zero imaginary part in Eq. $(A-6)$ :

$$
\left(1+\varepsilon^{2}\right) \delta-\theta \varepsilon=0
$$

Substituting Eq. (A-7) into Eq. $(A-6)$ we have:

$$
\left(\frac{M_{11}^{k}}{M_{11}^{1 k}}\right)^{\max }=1+\frac{0}{\varepsilon^{2}+1}
$$

The terms $\varepsilon$ and $\delta$ are not independent as both contain $w$. The term $r=(\omega k / \omega i)^{2}$ will be taken as the independent variable.

Rearranging Eq. $(A-7)$, we have

$$
B E=\theta\left(\frac{\varepsilon^{2}}{1+\varepsilon^{2}}\right)
$$

For $\epsilon^{2} \gg i$ or results insensitive to $\theta$,

$$
\delta E=0
$$


Substituting the definition of $\varepsilon, \delta$, and $\theta$ into $E q .(A-10)$, we have

$$
\frac{1}{\gamma^{k}}\left(\frac{\omega}{\omega^{k}}-\frac{\omega^{k}}{\omega}\right) \frac{1}{\gamma^{i}}\left(\frac{\omega}{\omega^{i}}-\frac{\omega^{i}}{\omega}\right)=\frac{\omega^{i} \bar{m}^{i}\left(\phi_{1}^{k}\right)^{2}}{\omega^{k} m^{k} \gamma^{i} \gamma^{k}}
$$

Rearranging Eq. (A-11) we obtain

$$
\left[\left(\frac{\omega}{\omega^{i}}\right)^{2}-\left(\frac{\omega^{k}}{\omega^{i}}\right)^{2}\right]\left[1-\left(\frac{\omega^{i}}{\omega}\right)^{2}\right]=\frac{\bar{m}^{i}\left(\phi_{1}^{k}\right)^{2}}{m^{k}}
$$

Let

$$
\Omega \equiv\left(\frac{\omega}{\omega^{i}}\right)^{2}, \quad r \equiv\left(\frac{\omega^{k}}{\omega^{i}}\right) \text { and } \beta=\frac{\bar{m}^{i}\left(\phi_{1}^{k}\right)^{2}}{m^{k}}
$$

Then

$$
\Omega^{2}-(r+\beta+1) \Omega+r=0
$$

Solving for $\Omega$, we obtain

$$
\Omega=\frac{r+\beta+1 \pm \sqrt{(r+\beta+1)^{2}-4 r}}{2}
$$

From the definition of $\varepsilon$ and $\Omega$,

$$
\varepsilon=\sqrt{\Omega} \cdot \frac{1}{\sqrt{\Omega}}
$$


Finally, from Eqs. $(A-1)$ and $(A-8)$,

$$
\frac{\left(\dot{u}_{1}^{k}\right)^{\max }}{\left(\dot{u}^{i k}\right)^{\max }}=1+\frac{\theta}{\varepsilon^{2}+1}
$$

Equation $(A-16)$ is the required expression of the ratio $\mathscr{R}$. Figure $A-1$ shows the ratio $R$ as a function of $r$ for $\gamma^{i} / \gamma^{k}=1$ and several $\bar{\gamma}$ and $\beta$. Figure A-2 shows the ratio $R$ of $r$ for $\bar{\gamma}=0.02$ and several $\beta$ and $\gamma^{i} / \gamma^{k}$. Figure A-3 shows the ratio $: R$ as a function of $\theta^{1}$ for $r=1$ and several $\gamma^{i} / \gamma^{k}$ and $\bar{\gamma}$.

Those curves show that, except for the tuned case $\omega^{k}=\omega^{i}$, the ratio $\mathscr{R}$ remains relatively constant, slightly higher than unity; i.e., the amplitude of the unloaded interface acceleration is only slightly higher than that of the loaded interface. 

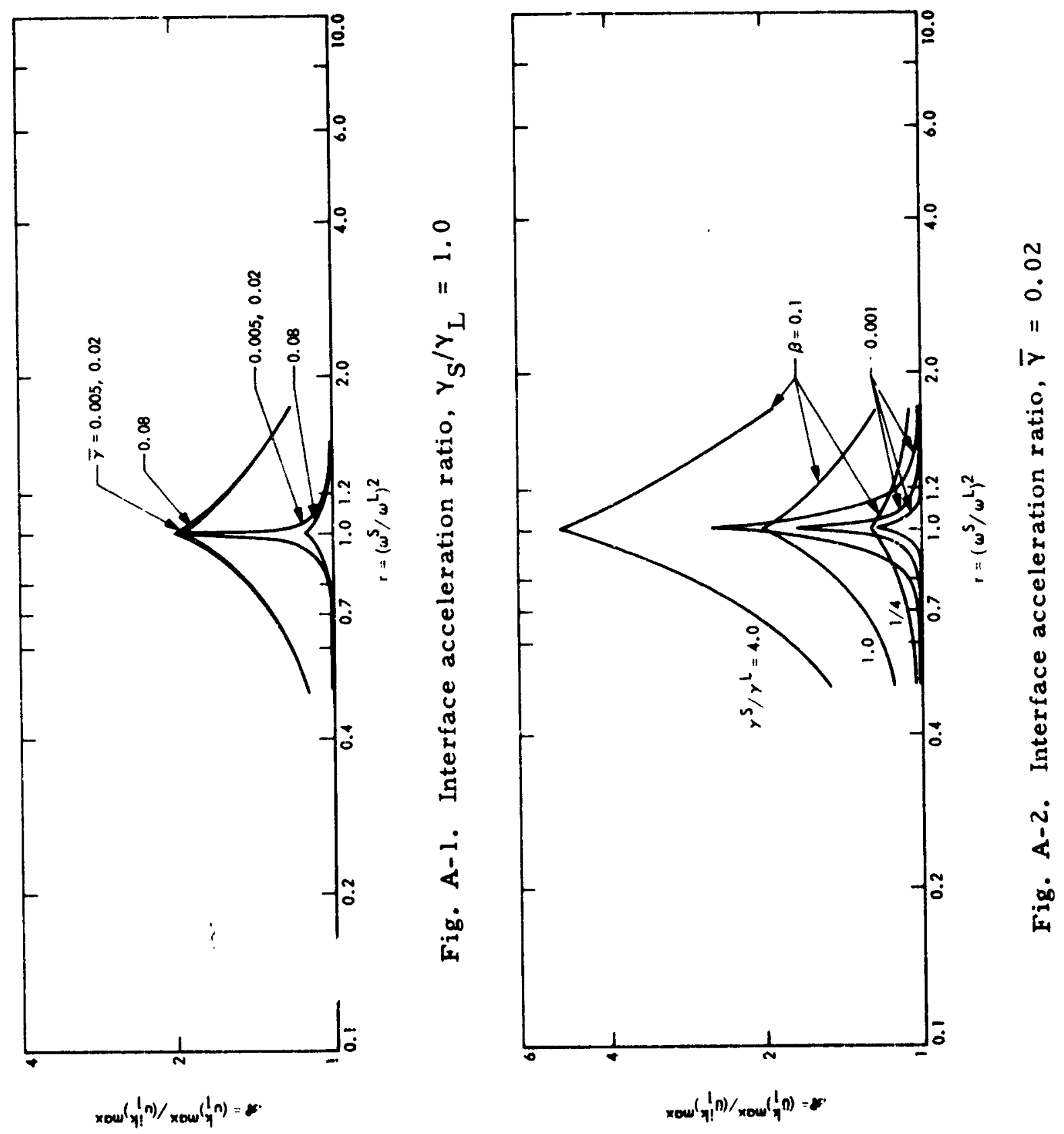


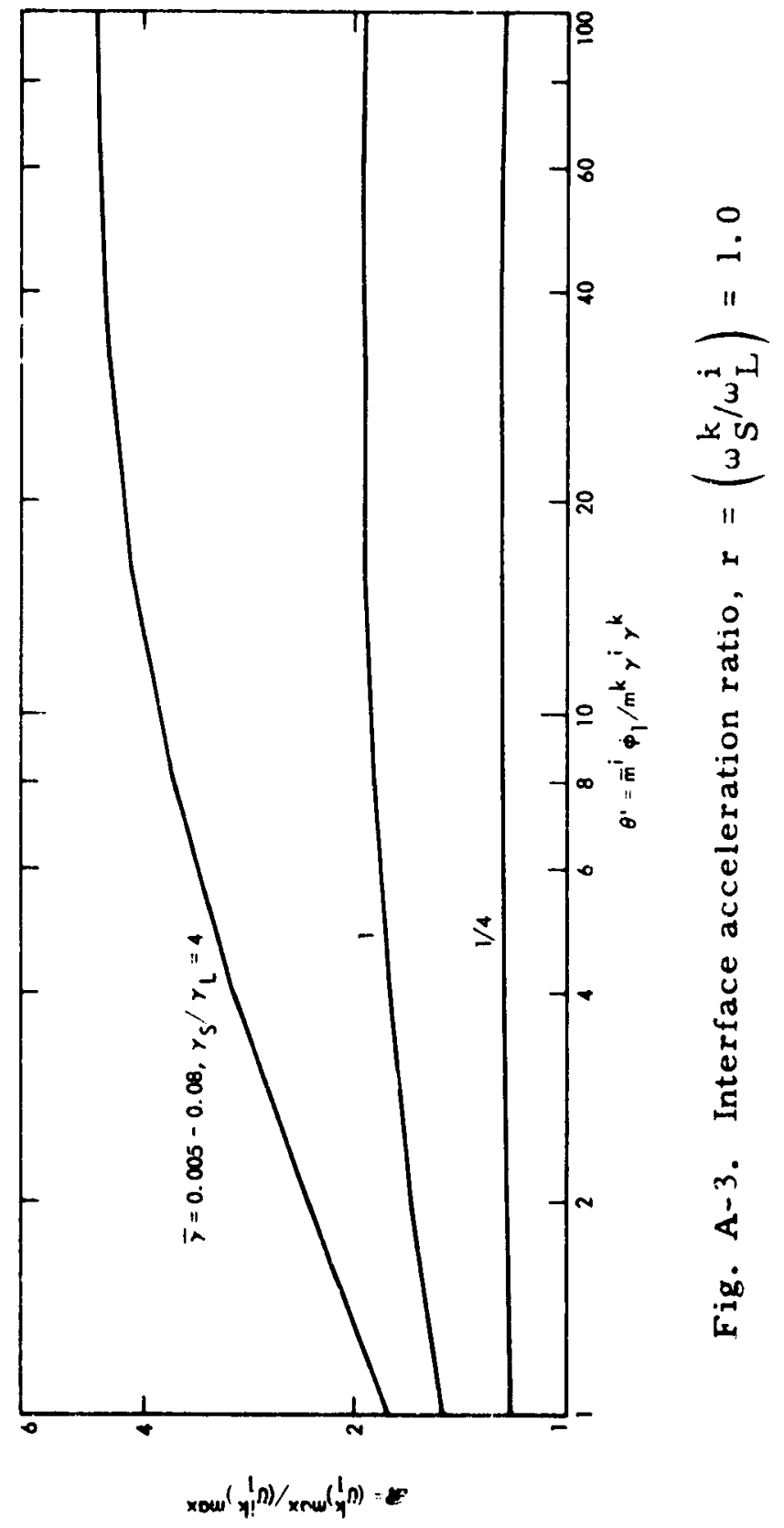




\section{APPENDIX B}

\section{CONSERVATIVE REPLACEMEINT FOR \\ REI.ATIVE DISPLACEMENT}

It will be shown that, given $C$,

$$
0.0<C<1.0
$$

The following inequality holds:

$$
u *+C\left(\left|u_{i}^{0}\right|-u^{*}\right)=\left|u_{i}^{0}+C\left(u_{i}^{0}-u_{i}^{0}\right)\right|
$$

Where the base motion $u_{1}^{\circ}$ is replaced by an upper bound $u^{*}$ in phase with $u_{i}^{\circ}$,

$$
u^{*} \geq\left|u_{i}\right|
$$

Two basic cases will be considered. The first provides for $u_{R}^{\circ}$, with the same sign as $u_{j}^{\circ}$; the second provides for $u_{R^{\circ}}^{\circ}$, with an opposite sign to $u_{i}^{\circ}$. For convenience, $u_{1}^{\circ}$ will be assumed positive. This is done without loss of generality by selection of an appropriate coordinate system. Let us call $u_{R}^{R}$ the reduced relative displacement

$$
u_{R}^{R} \equiv C\left(u_{i}^{0}-u_{i}^{\circ}\right)
$$

Case 1: $u_{R}^{R}>0$

$$
\text { If } u_{R}^{R}>0 \text { then } u_{R}^{0}>0
$$

If $u_{R}^{\circ}$ and $u_{i}^{\circ}$ are both $>0$, then $u_{i}^{\circ}$ is $>0$.

The absolute value signs in the inequality to be proved can be removed:

$$
u *+C\left(u_{i}^{0}-u *\right) \stackrel{?}{\geq} u_{i}^{0}+C\left(u_{i}^{0}-u_{i}^{0}\right)
$$


Simplifying and rearlanging, we obtain

$$
(1.0-C)\left(u *-u_{i}^{0}\right) \stackrel{?}{\geq} 0
$$

Since both factors are positive by definition, the inequality is proven in Case 1.

CASE 2: $\mathrm{u}_{\mathrm{R}}^{\mathrm{R}}<0$

If $u_{R}^{R}<0$, then $u_{R}^{\circ}<0$

Case 2 will be further subdivided into two subcases.

SUBCASE A: $u_{i}^{0} \geq u_{R}^{R}$

If $u_{i}^{0} \geq u_{R}^{R}$, the absolute value on the right side of the inequality to be proved may be removed.

$$
u *+C\left(\left|u_{i}^{0}\right|-u *\right) \stackrel{?}{\geq} u_{i}^{0}+C\left(u_{i}^{0}=u_{i}^{0}\right)
$$

If the inequality holds with the absolute value removed, which is Case 1 , the inequality is $p$ :oven in case $2 \mathrm{~A}$.

SUBCASE B: $u_{i}^{i}<u_{R}^{R}$

If $u_{i}<u_{R}^{R}$ the absolute value sign on the right side of the inequality to be proved may be removed after changing the sign of the terms included.

$$
u *+C\left(\left|u_{i}^{0}\right|-u^{*}\right) \stackrel{?}{\geq}-u_{i}^{0}-C\left(u_{i}^{0}-u_{i}^{0}\right)
$$

Rearranging, we obtain

$$
\left(u_{i}^{0}+u^{*}\right)(1-C)+C\left(\left|u_{i}^{0}\right|+u_{i}^{0}\right) \stackrel{?}{\geq} 0
$$

Both factors of both terms are positive by definition, so the inequality holds in Subcase 2B. 